\title{
PROCESS MAPPING FOR SERVICE DELIVERY THROUGH NSDG IN PUBLIC TRANSPORTATION IN INDIA
}

\author{
Sanjay K. Dwivedi and Ajay Kumar Bharti \\ Department of Computer Science, Babasaheb Bhimrao Ambedkar University, Lucknow, India
}

Received 2014-02-22; Revised 2014-03-12; Accepted 2014-05-12

\begin{abstract}
The involvement of e-Governance and Information and Communication Technology (ICT) in service delivery has been affecting every facet of governance. The public transportation sector is also enhancing their administrative and service delivery mechanism using Information and Communication Technologies. The existing public transportations (especially buses under SRTC-State Road Transport Corporations) in India provide cataloging and transaction related information on their websites, which lags due to absence of integrated and interoperable approach. Introduction of National e-Governance Service Delivery Gateway (NSDG) through the government of India helps to interconnect various departments at central level state level and municipal level. Public transportation especially SRTC's of different state should exploit the functionalities of NSDG to provide interconnected network of public transportation in India. The paper elaborates process mapping of public transportation for effective incorporation of National e-Governance Service Delivery Gateway (NSDG) using Accuprocess modelling tool. Process mapping provides a greater understanding of the information flow between different SRTC's of India which provides integration and interoperability for public transportation.
\end{abstract}

Keywords:e-Governance, Public Transportation, Process Mapping, Process Modelling, Integrated Transportation

\section{INTRODUCTION}

The bus based public transport provides an alternate mode of transportation. It makes the most optimum use of the available road space and fossil fuel by transporting the maximum number of people per unit of road space. Public transportation sector in the developing countries like India carries more than $90 \%$ of passengers by buses and about $65 \%$ of freight (ITSR, 2013), even though the sector faces severe problems such as lack of infrastructure, comfortable buses, revenue leakages due to corruption and financial resources which restrict investment and upgradation of the existing transportation system. Moreover the lack of proper and effective planning in public transportation sector in India has led to rapid growth in cars and two wheeled motor vehicle which causes congestion on roads that slows down the bus services or public transportation, ultimately increases the operating cost and discourage the use of public transportations (Bharti and Dwivedi, 2011). An economical pressure and deficit budget pressurizes the public transport sector to improve operational effectiveness and efficient services. The public transport sector should use information and communication technology as a powerful tool to achieve operational effectiveness in their service delivery. Web technologies enabled the government and administration of public transportation to reduce efforts and costs for their services using e-Governance in the sector. Effective implementation of e-Governance in the public transport sector will be able to minimize the economic pressure and improve service delivery in the sector integrating various State Road Transport Corporations (SRTC's) under the State Transport Undertakings (STU) (Review, 2010-11). An integrated and interoperable approach for the service delivery in the public transportation has been discussed in (Bharti and Dwivedi, 2013) to enhance the system. Corresponding Author: Sanjay K. Dwivedi, Department of Computer Science, Babasaheb Bhimrao Ambedkar University, Lucknow, India 
Process mapping is an approach to design information or activity flow diagram which helps to understand a system. This study provides the process mapping to understand the integrated and interoperable approach in public transportation using NSDG. AccuProcess Modeler 3.0 tool is used to design the process model given in Fig. 3.

\section{PROCESS MODELLING OR PROCESS MAPPING}

The business process modeling is highly essential to an organization. An organisation can define and produce a software process by its own way, some activities may common to all software processes. The major issues for an organization are increasing efficiency and improving process effectiveness, as well as standardizing and balancing the business processes. A business process can be decomposed into several sub-processes, comprising of their own attributes, but simultaneously contributing to achieving the goal of the super business process (AccuProcess, 2013).

Indulska et al. (2009) suggest six different capability areas for process model such as strategic alignment, governance, method, IT, people and culture, which are required to establish, progress and mature BPM in organizations. Process modeling being an necessary component of BPM, we adopted the capability area definition to the more specific process modeling context as follows:

- Strategic alignment is the continual tight linkage of process modeling to organizational priorities and processes, enabling achievement of business goals

- Governance establishes relevant and transparent accountability and decision-making processes to align rewards and guide actions in process modeling

- Methods are the approaches and techniques that support and enable consistent process modeling actions and outcomes

- Information technology is the software, hardware and information management systems that enables and support process modeling activities

- People are the individuals and groups who continually enhance and apply their process modeling-related expertise and knowledge

- Culture is the collective values and beliefs that shape process modeling-related attitudes and behaviours

Feiler, Humphrey defined a software process (Feiler and Humphrey, 1993) "A software process model is an abstract representation of the architecture, design and flow of the process". A process model is a set of activities undertaken to manage, develop and maintain process map, which focus on the construction of process rather than product's output.

According to (Hunt, 1996) "Processes can be modeled with different, mostly graphical, techniques-one of the more popular is process maps. Process maps are a proven analytical, communication and management tool intended to help process participants understand real business processes, make improvements to them or to implement a new process-driven structure in order to renovate business processes".

Process maps provide additional information for the process contrary to simple flowcharts. It considers information such as time, resources, environment and outputs, for every activity in the flowchart. We add the information to support real understanding of the process. Process map also have all the necessary elements required for simulation, to further quantitative and qualitative measures and analysis of the process performance.

\section{EXISTING PUBLIC TRANSPORTATION FOR INTEGRATED COMMUNICATION}

In India, the State Road Transport Corporations (SRTC's) are geographically dispersed in all states in India, which are unable to support integration and interoperability across the SRTC's as in Fig. 1. Technically it is not feasible to transform all the SRTC's together, to integrate the services of public transportation in a centralized environment. Therefore for integration and interoperability between the SRTC's requires a messaging middleware such as National e-Governance Service Delivery Gateway (eGR, 2013), so that all communications or information are routed through the NSDG to facilitate commuter for interstate communications. NSDG controls messaging, communication, authentication, authorizations and security as well as interoperability between the applications or departments. NSDG is one of the central Mission Mode Project (MMP) under National e-Governance Plan (NeGP, 2013) in India to provide flawless e-Governance services to the people, Central, States and local Governments through various gateways in NSDG.

Integration of public transportation is a step towards nationalization of public transportation using National eGovernance Service Delivery framework. In 12th five year plan, government of India is also looking for the national transport policy for the entire country up to 2032 (Report, 2012-17). Therefore we propose to establish and setup a domain gateway (Domain, 2013) to provide integrated services for the specific needs of public transportation through NSDG as in Fig. 2. 


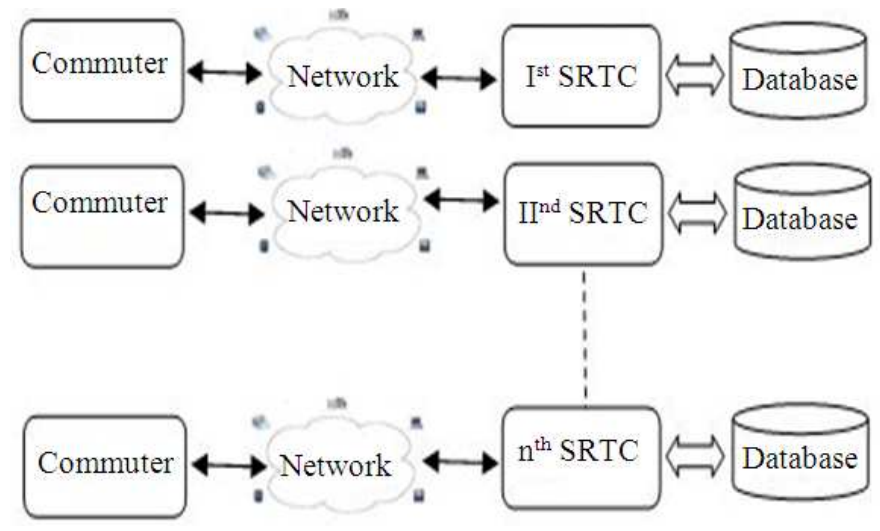

Fig. 1. Existing structure of the state road transport corpora-tion in India

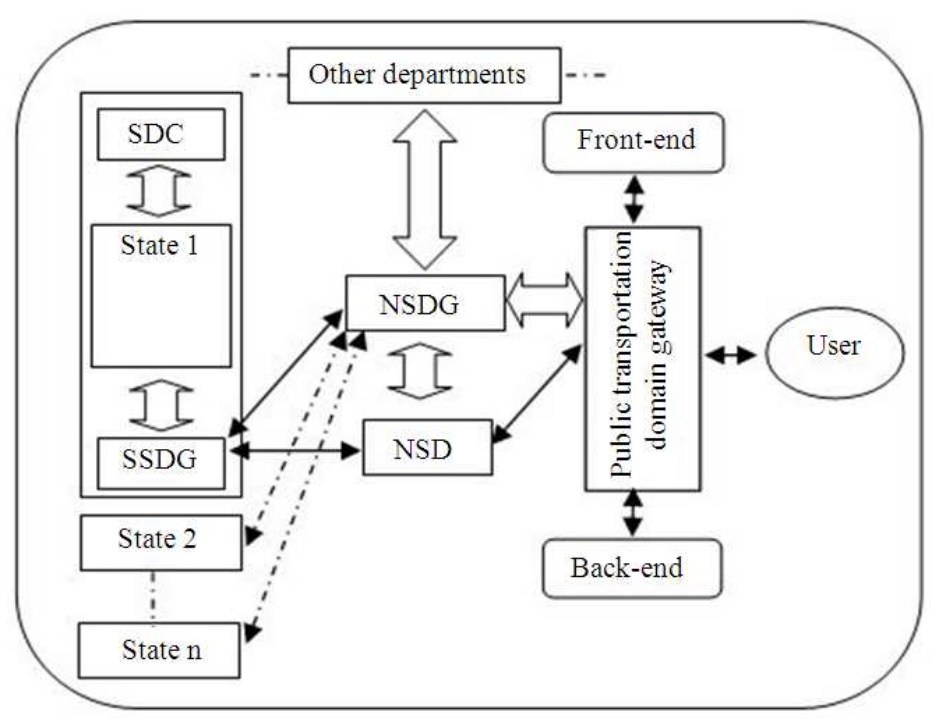

Fig. 2. Design for integration of SRTC's of different states

Public Domain gateway for public transportation will provides integrated and interoperable accessibility between SRTC's of the India. The model represents centralized access of information with distributed environment. Here we assume that all the states of India have agreed on common policy for the interstate communication to operate services in any states without any objection, because in India SRTC's comes under the State matter of concern. This restricts the interstate communication through the bus until SRTC's have an agreement for interstate operation. If the two state have an agreement to ply buses in between the two states. Suppose a user or commuter want to perform a two way journey between the states he has to check both websites of SRTC for to and fro journey separately. But integration between the SRTC will allow to access one stop services from any source state to destination state.

\section{PROCESS MAP FOR INTEGRATED SERVICES IN PUBLIC TRANSPORTATION IN INDIA}

Process mapping is away to design and visually present their business process through expressive graphical manner. AccuProcess Modeler 3.0 provides a drag and drop of element or activity to represents the flow of actual business process. 
Sanjay K. Dwivedi and Ajay Kumar Bharti / Journal of Computer Science 10 (10): 1955-1960, 2014

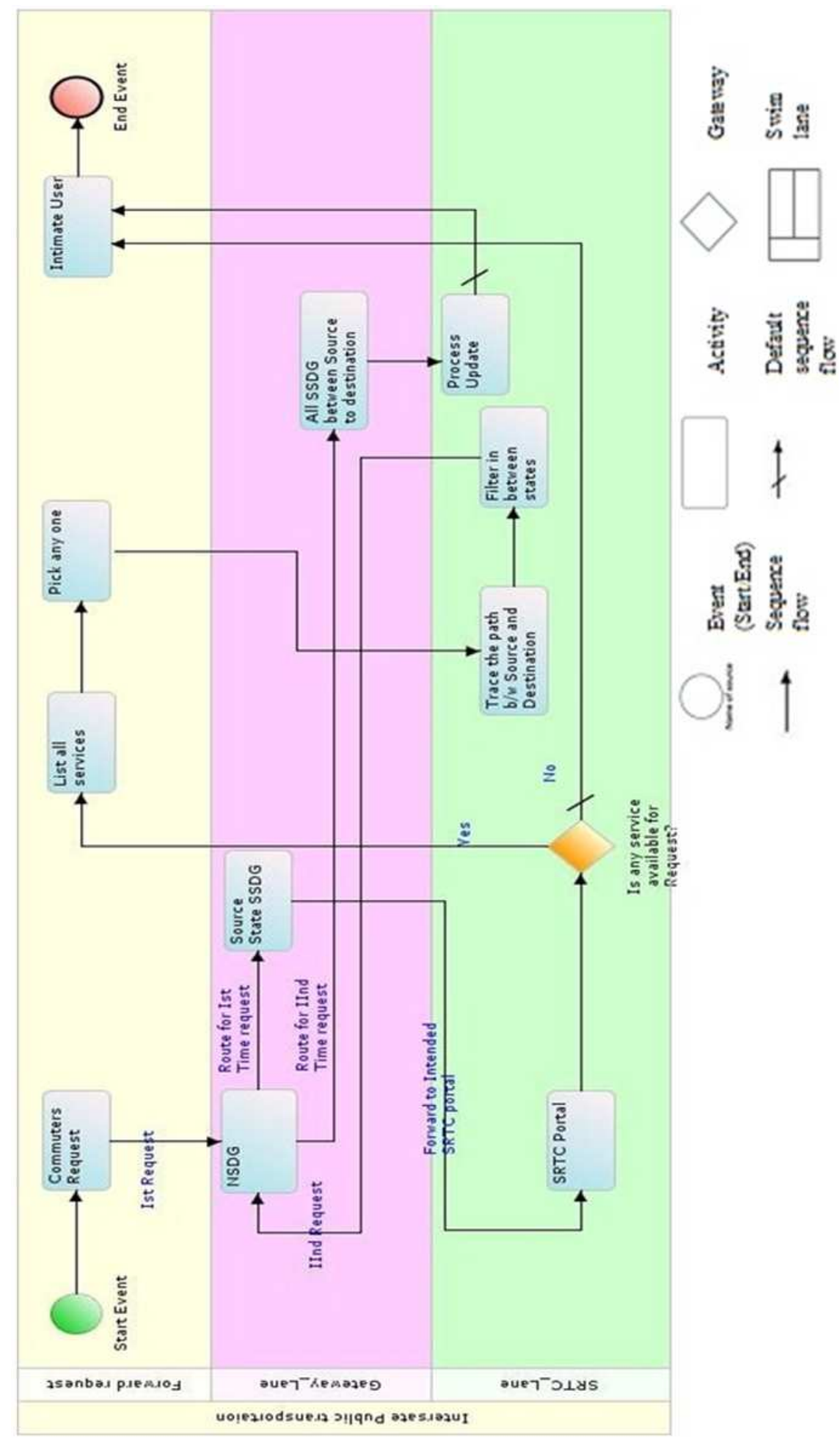

Fig. 3. Process model for integrated public transportation 
Process mapping in the Fig. 3 represents the graphical view of process map for the integrated approach of public transportation. The mapping represents the online reservation for the interstate communication using National e-Governance.

Service delivery gateway, where two or more states are involved in the transportation process. In the model NSDG and SSDG as a messaging gateway plays very important role for integrating the SRTC's. The process map shown in Fig. 3 will perform the following steps:

- Processes will starts when a user log on to the domain portal a interface fo the public transportation domain portal of SRTC

- A commuter submits their request in the desired format prescribed in the domain portal of SRTC and information passed to the NSDG with request id of the commuter

- Information is route to the source SSDG by NSDG for further processing

- The received information is passed to the specific state SRTC application portal for verification of the request

- $\quad$ SRTC verifies the request and forward accordingly, if SRTC provides any interstate services for the particular request it will list out for display to user. If availability of the services is not found according to the request intimate the user

- User has to select any one service form the list

- SRTC will take action on the commuter's choice of service and trace the path form source to destination for specific service

- Filter all unique state between the source and destination station in commuters request and forward the "list of states" to the NSDG with request id

- NSDG will route the specific request with request id to all the SSDG's coming in the "list of states"

- All the SSDG updates the information with particular request id in their databases

- Intimate the user for their request

- Terminate the process

\section{BENEFITS}

Process mapping of integrated public transportation as suggested in this study provides the flow of information and functionality of the sector and would provide the following benefits:

- The process models made by process maps are easily understandable without any special modelling
kn006Fwledge. It helps for clear representation of the flow between the activities performed by the elements of public transportation in the process map

- Process maps have all the necessary elements that are required for simulation of the public transportation for further analysis and performance measures for quantitative and qualitative analysis in the sector

- defined process in the public transportation, it also helps to decompose a major activity in to smaller one or sub process

- Activities of process maps provide additional information of the element of public transportation which is quite different from simple flowcharts

\section{CONCLUSION}

Process mapping is an approach to describe information or activity flow, which represents appropriate flow diagram for any system. Business process modelling using process mapping provides a clear view of process flow for the interstate public transportation using National e-Governance Service Delivery gateway. Process modelling tool provides the additional information about every activities of the process, flows and gateways used in mapping. This additional information enables the policy makers to design and develop projects in appropriate manner. In our process mapping it provides only information flow which cannot be used for analysis purpose due to lackness of quantitative data, but after appropriate changes it could be simulate by the modeling tool. In addition process modelling approach helps to analyse the existing process by the quantitative and qualitative evaluation of redesigned process through simulation.

\section{REFERENCES}

AccuProcess, 2013. AccuProcess Modeler User GuideBusiness Process Modeling.

Bharti, A.K. and S.K. Dwivedi, 2011. Design of an analytical and foresight based strategic model for eGovernance in public transportation. Proceedings of the 1st International Conference on Computational Intelligence and Information Technology, Nov. 7-8, 2011, Pune, India, pp: 615-620. DOI: 10.1007/9783-642-25734-6_105

Bharti, A.K. and S.K. Dwivedi, 2013, Integration of public transportation through national e-Governance service delivery framework. Int. J. Comput. Sci. 10: 189-192.

Domain, 2013. Domain gateway. Domain. 
Feiler, P.H. and W.S. Humphrey, 1993. Software process development and enactment: Concepts and definitions. Proceedings of the 2nd International Conference on Software Process, Feb. 25-26, IEEE Xplore Press, Berlin, pp: 28-40. DOI: 10.1109/SPCON.1993.236824

Hunt, D.V., 1996. Process Mapping: How to Reengineer your Business Process. 1st Edn., John Wiley and Sons, New York, ISBN-10: 0471132810 pp: 274.

ITSR, 2013. India Transport Sector-Roads.

Indulska, M., J. Recker, M. Rosemann and P. Green, 2009. Process modeling: Current issues and future challenges, advanced information systems engineering. Springer Link Lecture Notes Comput. Sci., 5565: 501-514.
NeGP, 2013. National e-Governance plan.

eGR, 2013. National e-Governance Service Delivery Gateway. eGovReach-A NASSCOM initiative.

Report, 2012/17. Report of the sub-group on SRTU under group on Road Transport Constituted by planning commission: 12th Five year plan 20122017.

Review, 2010-11. Review of the performance of state road transport undertakings. 